\title{
Priorities, barriers and facilitators for remote support of cancer symptoms: A survey of Canadian oncology nurses
}

by Gail Macartney, Dawn Stacey, Meg Carley and Margaret B. Harrison

\begin{abstract}
A survey of 368 Canadian oncology nurses revealed that $54 \%$ provided remote support to oncology patients. The most common symptoms identified were fatigue, pain, nausea, constipation, and anxiety. Frequent symptoms which nurses felt low confidence in managing were anxiety, neuropathy, depression, anorexia and skin alterations. Most nurses agreed that guidelines were needed that were accessible, and improved continuity of care. Forty-three per cent of respondents felt that existing guidelines were inadequate for complex symptoms. Only 54\% of respondents agreed they had received enough symptom management training. Common barriers included time constraints, obtaining accurate patient information via telephone, high workloads and knowledge or training deficits. Facilitating factors for provision of remote support included access to an electronic health record, accessible team members, available technology and ease of documentation.
\end{abstract}

\section{Background}

Telephone nursing advice services are growing both nationally and internationally to improve access to health care (Goodwin, 2007; Holmstrom \& Hoglund, 2007) and minimize costs (Wilson \& Hubert, 2002). With the growing cancer patient population and

\section{About the authors}

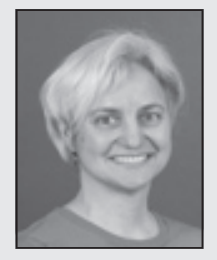

Gail Macartney, RN(EC), BScH, MSc(A), Nurse Practitioner, Division of Neurosurgery, Children's Hospital of Eastern Ontario, 401 Smyth Road, Ottawa, ON K1H $8 L 1$

Doctoral Student, Queen's University School of Nursing, 78 Barrie Street, Kingston, ON K7L $3 N 6$

E-mail: gail.macartney@sympatico.ca; Work: 613 737-7600 ext. 2640; Fax: 613 738-4228

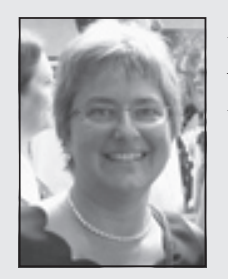

Dawn Stacey, RN, PhD, Director, Patient Decision Aids Research Group, Ottawa Hospital Research Institute, Associate Professor School of Nursing, University of Ottawa, Ottawa, ON

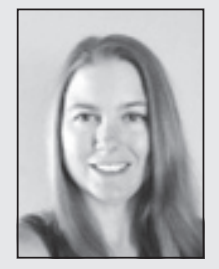

Meg Carley, BSc, Data Manager, Queen's University School of Nursing, 78 Barrie Street, Kingston, ON K7L 3N6

Data Manager, Canadian Partnership Against Cancer

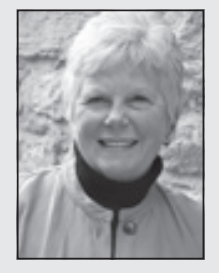

Margaret B. Harrison, RN, PhD, Professor, School of Nursing, Community Health and Epidemiology, Director, Queen's Joanna Briggs Collaboration, Senior Scientist Practice and Research in Nursing (PRN) Group, Queen's University, 78 Barrie Street, Kingston ON K7L 3N6 stress on cancer care resources, oncology nurses are faced with increased demands with respect to remotely supporting individuals and families to manage symptoms at home. A recent satisfaction survey conducted with 276 ambulatory cancer patients at an urban university teaching hospital in Montreal, Quebec, found that telephone contact with health professionals rated the lowest levels of patient satisfaction (Richard et al., 2010). Yet ability for patients to speak to professionals by telephone regarding their health concerns was identified as a priority. Clearly, it is important to better understand these health concerns and determine how competent nurses feel in dealing with them remotely.

Oncology nurses typically provide remote support over the telephone. Various terms used to describe the provision of healthrelated consultation by a professional over the phone include telehealth, telephone advice services, telenursing, telephone consultation, teletriage and telephone-mediated care. Telephone-mediated care is defined as the provision of information, advice or support (Wilson \& Hubert, 2002), whereas, teletriage is the process of screening and collecting a caller's symptoms over the telephone to evaluate the urgency of a health problem (Black, 2007; Stacey, 2003).

Symptom assessment and management is a particularly challenging task when the nurse-patient interactions occur over the phone, given that care providers are challenged to accurately assess a situation, with limited sensory input (Black, 2007). Oncology nurses are required to ascertain enough accurate information pertaining to particular symptoms, to identify if self-care management strategies will suffice or whether the patient requires a more thorough assessment in person. The provision of remote support can enhance more appropriate health care utilization (Mayo, Chang \& Omery, 2002). Although common symptoms experienced by ambulatory oncology patients have been reported in other studies (Barbera et al., 2010), little is known about which symptoms patients report through telephone-based healthcare services. The purpose of this study was to identify the most prevalent and challenging symptom management issues for oncology nurses providing remote symptom support. Additionally, facilitating factors and potential barriers to the provision of remote support for symptom assessment, triage and management were explored.

\section{Methods}

Design. An online survey was conducted between September 3, 2009, and October 27, 2009, of oncology nurses across Canada. Ethics approval was obtained from the Queen's University Research Ethics Board.

Instrument. The survey tool was developed based upon a previous survey of telephone nursing services offered through ambulatory oncology programs in Ontario (Stacey et al., 2007), previous surveys of nurses' use of evidence-based tools in clinical practice (e.g., leg ulcer guidelines, patient decision aids) (Graham et al., 2001; Stacey et al., 2005), and the Ottawa Model of Research Use (Graham \& Logan, 2004). Survey items were modified to reflect oncology nursing and the ambulatory care setting. Nurses rated 16 common cancer treatment-related symptoms on a 10-point Likert scale from extremely rare to extremely common in terms of symptom frequency. Nursing confidence was rated for the same symptoms on a 10-point scale from not confident to extremely confident. Additional symptoms to the 16 were captured in an open-ended question. The survey included 19 statements that measured nurses' attitude, behaviours, 
knowledge, confidence and perceptions of environmental barriers to guideline use. Respondents rated each statement on a five-point Likert scale ranging from strongly agree to strongly disagree. Openended questions asked participants to identify barriers and facilitators to the provision of remote symptom management. The survey was also designed to capture information pertaining to how and when remote support was provided including infrastructure characteristics in the clinical setting such as access to guidelines and electronic documentation. Face validity of the survey questionnaire was established by a group of 11 researchers and practitioners with expertise in oncology nursing and research methods. The online survey was pre-tested with three oncology nurses.

Participants. Registered nurses who could read English and were members of the Canadian Association of Nurses in Oncology (CANO), Cancer Care Ontario's Advanced Practice Nurses Community of Practice, and/or members of a Canadian Oncology Nurse Educators list serve were invited to participate.

\section{Table 1: Characteristics of remote support responders}

\begin{tabular}{|l|c|}
\hline Responder Characteristics & Frequency (\%) $\mathbf{n = 1 9 7}$ \\
\hline Oncology setting for remote support & \\
Ambulatory cancer program & $121(61.4)$ \\
Adult inpatient & $23(11.7)$ \\
Homecare/community & $7(3.6)$ \\
Pediatric inpatient & $1(0.5)$ \\
Other (e.g. ADD) & $15(7.6)$ \\
\hline Professional affiliations* & \\
Member of CANO/ACIO & $140(71.1)$ \\
Member of advanced practice nursing COP & $21(10.7)$ \\
Canadian Certification (CON(C)) & $110(55.8)$ \\
American Certification (OCN) & $8(4.1)$ \\
\hline Academic credentials & \\
Diploma in Nursing & $104(52.8)$ \\
Baccalaureate in Nursing & $107(54.3)$ \\
Other Baccalaureate & $13(6.6)$ \\
Master's in Nursing & $50(25.4)$ \\
Other Master's & $11(5.6)$
\end{tabular}

Years as registered nurse

$>2-5$ years $4(2.0)$

$>5-10$ years

$13(6.6)$

$>10-15$ years

$14(7.1)$

$>15$ years

135 (68.5)

Years of oncology nursing experience

$<1-2$ years

$>2-5$ years

$>5-10$ years

$>10-15$ years

$>15$ years

3(1.5)

$18(9.1)$

27 (13.7)

37 (18.8)

$81(41.1)$

Current oncology nursing clinical practice*

Chemotherapy $108(54.8)$

Supportive Care

$72(36.5)$

Palliative Care

Radiation Therapy

64 (32.5)

47 (23.9)

Survivorship

26 (13.2)

Surgery

Prevention

$26(13.2)$

15 (7.6)

Screening

9 (4.6)

Rehabilitation

4 (2.0)
Procedures. The survey was administered in English as an online questionnaire using the survey software Survey Monkey ${ }^{\circledR}$. Procedures for administering the survey online were based on the Tailored Design Method for Surveys (Dillman, 2000). Identified nurses received the initial invitation as an email that explained the purpose of the survey, instructions, deadlines, and expected time for completion. A link was provided to an information sheet that provided more details and access to the survey questionnaire. The survey took approximately 10 minutes to complete. As per the Dillman (2000) approach, reminders were sent out at two, four, and five weeks for a total of four contacts. Electronic reminders were automatically generated to non-responders. Data collected in Survey Monkey ${ }^{\circledR}$ were downloaded into IBM $\subset$ SPSS $\subset$ (Version 19 for Windows).

Analysis. Demographic results were tabulated and described using univariate statistics. Data were described as frequency (per cent) for categorical variables, and mean, standard deviation (SD), range, median for continuous variables. To identify symptom priorities, symptoms were rank ordered from high to low frequency (from 1 to 16) and for low to high nursing confidence (from 1 to 16). Nursing

Table 2: Characteristics of remote support provision

\begin{tabular}{|c|c|}
\hline & $\begin{array}{l}\text { Frequency (\%) } \\
n=197\end{array}$ \\
\hline $\begin{array}{l}\text { Available hours for patients to make contact } \\
\text { During regular hours (Monday to Friday } \\
\text { daytime) } \\
24 \text { hrs a day, } 7 \text { days a week } \\
\text { Weekends \& holidays } \\
\text { Evenings }\end{array}$ & $\begin{array}{l}175(88.8) \\
11(5.6) \\
6(3.0) \\
2(1.0)\end{array}$ \\
\hline $\begin{array}{l}\text { Model of care for remote support services } \\
\text { Primary nurse model } \\
\text { Nurse assigned to manage all calls for the } \\
\text { day/shift } \\
\text { Other (e.g. team nursing) }\end{array}$ & $\begin{array}{l}92(46.7) \\
41(20.8) \\
55(27.9)\end{array}$ \\
\hline $\begin{array}{l}\text { Documentation of calls including e-mails in } \\
\text { health record } \\
\text { Yes, routinely } \\
\text { Yes, as necessary } \\
\text { No }\end{array}$ & $\begin{array}{c}124(62.9) \\
49(24.9) \\
15(7.6)\end{array}$ \\
\hline $\begin{array}{l}\text { Documentation format } \\
\text { Paper-based forms } \\
\text { Computer-based documentation } \\
\text { Other }\end{array}$ & $\begin{array}{c}107(54.3) \\
51(25.9) \\
16(8.1)\end{array}$ \\
\hline $\begin{array}{l}\text { Use of clinical protocols/guidelines for } \\
\text { triaging or managing symptoms } \\
\text { Yes } \\
\text { No }\end{array}$ & $\begin{array}{c}134(68.0) \\
36(18.3)\end{array}$ \\
\hline $\begin{array}{l}\text { Use of clinical practice protocols/guidelines } \\
\text { Used as a desk top reference } \\
\text { Used primarily for orientation of nurses } \\
\text { Integrated computer-based system } \\
\text { Not used } \\
\text { Other }\end{array}$ & $\begin{array}{c}132(67.0) \\
51(25.9) \\
41(20.8) \\
15(7.6) \\
16(8.1)\end{array}$ \\
\hline $\begin{array}{l}\text { Source of protocols/guidelines } \\
\text { Cancer Care Ontario guidelines } \\
\text { Developed own } \\
\text { B.C. Cancer Agency guidelines } \\
\text { Other }\end{array}$ & $\begin{array}{l}96(48.7) \\
47(23.9) \\
42(21.3) \\
35(17.8)\end{array}$ \\
\hline
\end{tabular}


confidence from low to high was plotted against symptom frequency from low to high for each symptom. Responses to the Likert scale attitudes, behaviours and knowledge questions were reclassified into agree (strongly agree or agree), disagree (strongly disagree and disagree), and neutral. Qualitative thematic content analysis was used for open-ended questions about other symptoms and barriers to the provision of remote support. Additional symptoms were categorized as physical, emotional/coping, cognitive, personal, logistic and other general issues.

\section{Results}

Of the 653 potential respondents, $56 \%(n=368)$ completed the survey and of those, $54 \%(197 / 368)$ provided remote support for oncology patients [98\% (193/197) by telephone; 30\% (60/197) by email]. The typical respondent worked in an ambulatory oncology program, was a member of CANO, was a certified oncology nurse, had a Baccalaureate degree, and had worked in nursing for more than 15 years (Table 1). Nurses' clinical practice focused on chemotherapy administration (55\%), supportive care (37\%), palliative care (32\%) and radiation therapy (24\%). Of the 197 respondents who provided remote support, $89 \%$ identified that support was provided during regular working hours (from Monday to Friday) and 10\% provided remote support outside regular working hours (e.g., weekends, evenings, 24 hours per day, seven days per week coverage) (see Table 2). The majority of respondents routinely documented calls with 54\% using paper-based forms and 8\% denied documenting remote encounters anywhere in the health record. Two-thirds of nurses used protocols or guidelines, but primarily as a desktop reference (67\%). Guidelines used were from Cancer Care Ontario (CCO) (49\%), British Columbia (21\%) or developed by their own institution (24\%).
Priorities for remote symptom management. The five most frequently reported symptoms were: fatigue (most common), pain, nausea, constipation, and anxiety (least common) (Figure 1). Nurses felt least confident managing, in order of increasing confidence: depression, dysuria/hematuria, anorexia, breathlessness and neuropathy; but were most confident with managing fever, constipation, nausea, vomiting and diarrhea. The priority symptoms based on high frequency and low nursing confidence included anxiety, neuropathy, depression, anorexia and skin alterations. Respondents identified additional issues patients identified through remote nursing support services. Physical issues included swelling, rashes, bleeding, dizziness, sleep disturbances, cough and xerostomia. Emotional challenges included dealing with anger, distress, or coping with terminal illness. Cognitive issues included confusion and steroid induced mood alterations. Intimacy, sexuality and body image issues were also identified.

Nursing attitudes, behaviours, knowledge and confidence. Of at least 171 respondents, most nurses agreed that guidelines: were needed (89\%), promoted continuity (93\%) and improved patient care (89\%) (Table 3). Eighty-six nurses (49\%) agreed that there was an expectation from supervisors to use guidelines. Most nurses agreed that guidelines were consistent with physician practice (46\%) and supported by the multidisciplinary team (60\%). Most nurses had easy access to guidelines (70\%), used guidelines to manage symptoms (69\%), could easily document guideline use (69\%) and provided written information to enhance patient education (76\%). Most nurses did not feel that their clinical judgment was constrained by guidelines (68\%); however, 121 nurses (69\%) felt confident in their abilities to manage symptoms without guidelines. Only 93 nurses (54\%) agreed that they had received enough symptom management training and

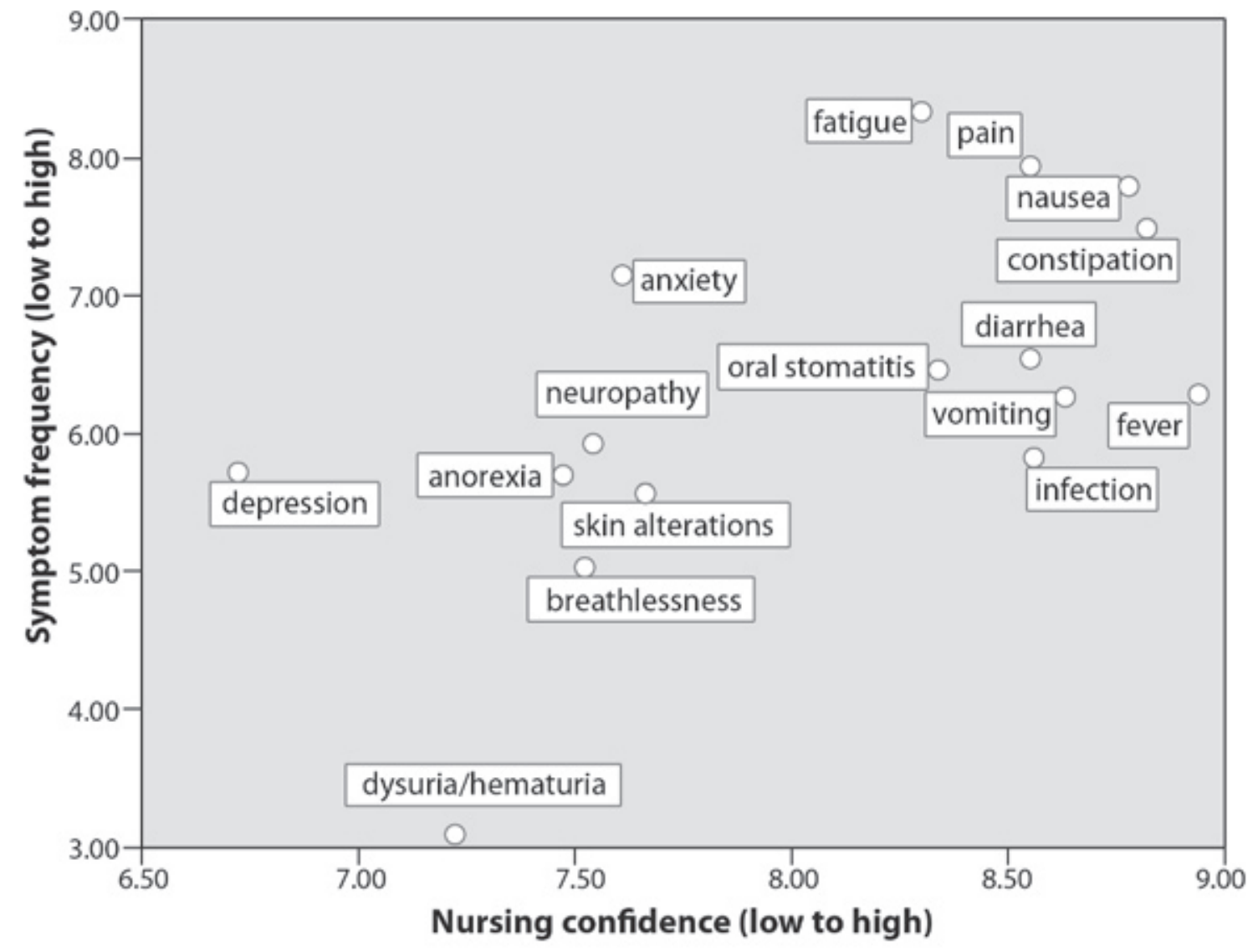

Figure 1: Nursing confidence and symptom frequency 
75 nurses (43\%) felt that current symptom management guidelines were inadequate for patients with multiple/complex symptoms. One hundred and seventeen nurses (68\%) agreed that electronic access to guidelines would increase their use. Sixty-two per cent of nurses did not feel under constant pressure for short patient interactions. Forty-four nurses (25\%) agreed that they did not have enough time to find and use guidelines.

Facilitators. Content analysis of open-ended responses revealed six key facilitators of remote support provision (Table 4). Many nurses identified that to facilitate use of symptom guidelines they needed guidelines that are accessible, electronic, evidence-based, and up to date. Remote support was facilitated by: a) access to an electronic health record with current health information; b) responsiveness of physician colleagues and multidisciplinary team members; c) administrative support; and d) available, user-friendly computers with a documentation system that was easy to use, standardized, electronic and formatted for remote support. Two respondents identified the need for medical directives to support prescribing medications required for symptom management.
Potential barriers. Content analysis revealed eight key barriers to the provision of remote support (Table 4). Fifty nurses (29\%) identified time constraints as the most significant barrier. Thirty-six nurses (21\%) identified the challenge of getting accurate patient information over the phone as a significant barrier (e.g., not being able to see the patient, communication issues, accuracy of the information about the patient's past medical history and medications). Twenty-one (12\%) respondents identified heavy workload and inadequate staffing as challenges. Twenty nurses (11\%) identified issues related to nursing knowledge, experience, assessment skills and training. Fourteen respondents (8\%) identified inadequacy of access to support services including diagnostics, pharmacy, homecare, family physicians and emergency departments. Five respondents (3\%) described difficulty using existing guidelines due to the fact that multiple, complex symptoms did not fit existing single symptom guidelines. Two respondents (1\%) identified environmental challenges including noisy environments and inconsistent physician practices.

Table 3: Nurses' attitudes, behaviours, knowledge, confidence and perceptions of environmental factors affecting remote telephone support

\begin{tabular}{|c|c|c|c|c|}
\hline & $\mathbf{n}$ & Disagree (\%) & Neutral (\%) & Agree (\%) \\
\hline \multicolumn{5}{|l|}{ Nurses' attitude toward guidelines } \\
\hline Guidelines promote continuity & 175 & 2.8 & 4.6 & 92.6 \\
\hline Guidelines improve patient care & 175 & 2.3 & 8.6 & 89.1 \\
\hline Guidelines are needed & 173 & 2.9 & 8.1 & 89.0 \\
\hline Guidelines are supported by multidisciplinary team & 175 & 11.4 & 29.1 & 59.5 \\
\hline Supervisors expect me to use guidelines & 174 & 20.7 & 29.9 & 49.4 \\
\hline Guidelines are consistent with physician practice & 174 & 23.0 & 31.0 & 46.0 \\
\hline \multicolumn{5}{|l|}{ Nurses' behaviours } \\
\hline I provide written information to enhance patient education & 171 & 10.5 & 13.5 & 76.0 \\
\hline I have easy access to evidence-based guidelines & 175 & 11.4 & 18.3 & 70.3 \\
\hline I can easily document & 173 & 16.1 & 15.0 & 68.9 \\
\hline I use guidelines to manage symptoms & 173 & 11.0 & 20.2 & 68.8 \\
\hline \multicolumn{5}{|l|}{ Nurses' knowledge and confidence } \\
\hline I feel confident in my abilities without guidelines & 175 & 14.9 & 16.0 & 69.1 \\
\hline I received enough symptom management training & 173 & 26.0 & 20.2 & 53.8 \\
\hline Protocols are inadequate for multiple/complex symptoms & 175 & 24.0 & 33.1 & 42.9 \\
\hline I do not have adequate knowledge to manage symptoms & 174 & 92.0 & 4.6 & 3.4 \\
\hline My clinical judgment is constrained by guidelines & 174 & 67.8 & 28.8 & 3.4 \\
\hline I learned what I know from other nurses & 174 & 47.7 & 25.9 & 26.4 \\
\hline \multicolumn{5}{|l|}{ Nurses' perceptions of environmental factors } \\
\hline Electronic access would increase use & 173 & 8.1 & 24.3 & 67.6 \\
\hline I feel constant pressure for short interactions & 175 & 62.3 & 21.1 & 16.6 \\
\hline There is no time to find and use guidelines & 175 & 44.0 & 30.9 & 25.1 \\
\hline
\end{tabular}




\section{Discussion}

This is the first known study to assess oncology nurses' perceptions of symptom prevalence, confidence with remote symptom management, and factors influencing provision of remote support. Most respondents who provided remote support were experienced nurses with oncology expertise, as recognized by certification. Remote support was primarily provided by telephone during regular working hours from Monday through Friday and documented on paper-based formats in the health record. Cancer Care Ontario's guidelines were the most frequently used, but primarily as a desktop reference.

Although nurses reported having a strong knowledge, feeling confident with symptom management and having positive attitudes toward using symptom guidelines, they identified that accessible, electronic, updated, evidence-based guidelines were needed to better facilitate remote symptom support. Furthermore, nurses reported that access to patients' electronic health records with electronic documentation would further enhance use of symptom guidelines. These findings are consistent with our previous study that revealed that oncology nurses are more likely to document calls when they have access to electronic health records (Stacey et al., 2007). Accessible, easy to use resources and well-designed documentation tools facilitated optimal patient care (Black, 2007). Province-wide nursing call centre programs have protocols to guide nurses' decision-making and are supported by electronic documentation systems (Stacey et al., 2003). Although these resources are expected for quality telephone-based care (College of Nurses of Ontario, 2005), they are not consistently provided in Canadian oncology programs.

Concern has been expressed that use of guidelines and protocols threatens the caring aspect of nursing care (Wilson \& Hubert, 2002) and their application lacks recognition of individual patient preferences (Black, 2007; Stacey et al., 2007). However, nurses in this study showed positive attitudes toward using guidelines. This may be reflective of changing attitudes toward guidelines given increasing access to these types of evidence-based resources and seeing them as a tool. Or it could reflect response bias with nurses providing responses they considered more appropriate rather than reflecting current practice.
The most common barriers identified were high workload, time constraints, and significant concern regarding the challenge of acquiring accurate patient information over the telephone. High workload and time constraints are consistent barriers identified in any practice-changing initiative and are difficult to address on their own (Conley et al., 2010). Accuracy of patient information during telephone-mediated symptom support has innate issues given limited sensory input (Black, 2007). Nurses in this study identified factors that could mediate these barriers. For example, they expressed great difficulty not being able to understand patients' or their family members' descriptions of the issue of concern. Language barriers, unclear speech or difficulties hearing clients while in their noisy work environments exacerbated these situations. These challenges could be minimized by use of standardized assessment tools, access to up-to-date patient records available electronically and provision of quiet workspaces, as described by nurses in this study. As well, training in telephone-mediated nursing practice has the potential to develop alternative ways of assessing symptom severity when limited to hearing only (Black, 2007; Stacey et al., 2007).

The need for further training was reflected in the qualitative feedback from the experienced oncology nurses surveyed. Interestingly, there is no consensus in the literature on what skills nurses providing telephone triage should have. For example, Black (2007) proposes that nurses require a minimum of three to five years of varied oncology nursing experience along with above average interpersonal skills and communication skills. Conley and colleagues (2010) recommend a set level of competency for all oncology nurses who participate in the telephone triage role with standardized evidencebased approach to patient assessment, follow-up and documentation. In a qualitative study exploring the ethical challenges of nurses providing telephone nursing, Holmstrom and Hoglund (2007) found that autonomy, integrity and prioritization were the important issues. Furthermore, building ethical competence was identified as potentially helping to foster reduced moral uncertainty and distress among telenurses. Nurses in this study highlighted the need for training in use of symptom guidelines and multiple symptom management (Holmstrom \& Hoglund, 2007).

\begin{tabular}{|c|c|}
\hline Barriers & Facilitators \\
\hline $\begin{array}{l}\text { Time (50) } \\
\text { - Time constraints } \\
\text { Challenge of acquiring accurate information (36) } \\
\text { - Challenge of getting accurate information about symptom (11) } \\
\text { - Not being able to see patient (10) } \\
\text { - Communication challenges: language, hearing, speech (8) } \\
\text { - Information needed about past medical history, medications (5) } \\
\text { - Not knowing patient (2) } \\
\text { Workload (21) } \\
\text { - Heavy workload, inadequate staffing } \\
\text { Nursing attributes (20) } \\
\text { - Lack of knowledge, experience, assessment skills (12) } \\
\text { - Lack of training (8) } \\
\text { Support services (14) } \\
\text { - Need access to diagnostics, pharmacy, homecare, family } \\
\text { physician, ER } \\
\text { Symptom Complexity (5) } \\
\text { - Multiple and complex symptoms do not fit guidelines } \\
\text { Environment (2) } \\
\text { - Noisy } \\
\text { Team Attributes (2) } \\
\text { - Inconsistent practice } \\
\text { - Unique MD preferences }\end{array}$ & $\begin{array}{l}\text { Access to Symptom Guidelines (22) } \\
\text { - Accessible, electronic, up to date, evidence-based (18) } \\
\text { - Patient education materials needed to support (3) } \\
\text { - Available in French (1) } \\
\text { Electronic Health Record (16) } \\
\text { - Accessible } \\
\text { - Includes current documentation, test results } \\
\text { Team Attributes (11) } \\
\text { - MD availability, responsiveness, support } \\
\text { - Multidisciplinary team support } \\
\text { - Administrator support } \\
\text { Technology-computers (9) } \\
\text { - Accessible, available, user-friendly } \\
\text { Documentation (7) } \\
\text { - Easy to use } \\
\text { - Adequate time to complete } \\
\text { - Standardized } \\
\text { - Formatted for telephone support } \\
\text { - Electronic } \\
\text { Model of Care (3) } \\
\text { - Primary nursing model } \\
\text { Medical Directives (2) } \\
\text { - Needed to facilitate prescription of symptom management } \\
\text { medications } \\
\text { Number of comments in parentheses }\end{array}$ \\
\hline
\end{tabular}


Current guidelines are inadequate for nurses caring for oncology patients experiencing multiple and complex symptoms. The most common guidelines identified as being used by oncology nurses are paper-based guidelines and, thereby, are limited to presenting single symptoms. Their use with multiple symptoms requires nurses to identify the most severe or bothersome symptom to select the single symptom protocol. However, people with cancer presenting with multiple symptoms may not be experiencing interrelated symptoms and, as such, require a more comprehensive approach to symptom management (Barsevick, 2007; Miaskowski, Dodd \& Lee, 2004). A key issue is that existing guidelines do not address how multiple symptoms may share common etiologies or how addressing one symptom such as pain may also positively impact another symptom such as sleep. Nurses are not specifically taught how to triage symptom severity when multiple symptoms coexist. Limitations to implementing standardized protocols include the fact that patients do not always fit into predetermined algorithms, minimizing the patient-specific aspects of a situation (Black, 2007).

Several limitations have been identified in this study. The generalizability of these results should be taken in context. Most respondents in this study were experienced oncology nurses working in a public health care system. Responses, therefore, may not reflect the views of more novice oncology nurses or those working in nonCanadian health care settings. Secondly, given some inconsistent responses between the quantitative and the qualitative responses, it is possible that some nurses provided responses they thought were the "right" answers rather than reflecting their own perspectives.

\section{REFERENCES}

Barbera, L., Seow, H., Howell, D., Sutradhar, R., Earle, C., Liu, Y., et al. (2010). Symptom burden and performance status in a population-based cohort of ambulatory cancer patients. Cancer, 116(24), 5767-5776.

Barsevick, A.M. (2007). The elusive concept of the symptom cluster. Oncology Nursing Forum, 34(5), 971-980.

Black, K.L. (2007). Standardization of telephone triage in pediatric oncology. Journal of Pediatric Oncology Nursing, 24(4), 190-199.

College of Nurses of Ontario Practice Guidelines. (2005). Telepractice. Ottawa: College of Nurses of Ontario, Pub. No. 41041.

Conley, S.B., O’Hanlon Curry, J., Hines, M., Baker, K., Schmidt, K., Zwier, K., Siever B., ... Kline, N.E. Association of Pediatric Hematology/Oncology Nursing. (2010). Consensus statements: Ambulatory pediatric oncology nursing practice. Journal of Pediatric Oncology Nursing, 27(1), 6-9.

Dillman, D.A. (2000). Mail and Internet surveys: The tailored design. New York: John Wiley \& Sons.

Dodd, M., Janson, S., Facione, N., Faucett, J., Froelicher, E. S., Humphreys, J., et al. (2001). Advancing the science of symptom management. Journal of Advanced Nursing, 33(5), 668-676.

Dodd, M.J., Miaskowski, C., \& Lee, K.A. (2004). Occurrence of symptom clusters. Journal of the National Cancer Institute, Monographs(32), 76-78.

Goodwin, S. (2007). Telephone nursing: An emerging practice area. Nursing Leadership, 20(4), 37-45.

Graham, I., Harrison, M.B., Moffat, C., \& Franks, P. (2001). Leg ulcer care: Nursing attitudes and knowledge. The Canadian Nurse, 97(3), 19-24.

Graham. I., \& Logan, J. (2004). Innovations in knowledge transfer and continuity of care. Canadian Journal of Nursing Research, 36, 89-103.

\section{Conclusion}

Experienced oncology nurses surveyed in this study provided primarily remote telephone support for symptom management during regular working hours using symptom guidelines, as a desktop reference. Nurses had positive attitudes toward having access to evidence-based, up-to-date guidelines but identified that to be useful, they needed electronic applications linked into documentation on the patients' electronic health record. Clinical priorities for guideline developers include symptom management of anxiety, neuropathies, anorexia, depression, and skin alterations. Additionally, guidelines and training are needed to assist nurses in the identification, prioritization and management of concurrent symptoms. Symptoms that cluster may share common underlying etiologies and benefit from similar management strategies. In addition to time constraints and workload, nurses identified the challenges of obtaining accurate patient information over the phone. Further research is needed to standardize criteria for remote patient assessment and evaluate the empiric rigor of guidelines used in remote symptom management. Additionally, there is a need to link the use of standardized, evidence-based guidelines to positive patient care outcomes, as very little research exists to date (Mayo et al., 2002; Wilson \& Hubert, 2002).

\section{Acknowledgements and funding}

This survey was conducted as one of several studies from the pan-Canadian Oncology Symptom Triage and Remote Support (COSTaRS). The Canadian Partnership Against Cancer (the Partnership) provided funding to support this project.

Holmstrom, I., \& Hoglund, A.T. (2007). The faceless encounter: Ethical dilemmas in telephone nursing. Journal of Clinical Nursing, 16(10), 1865-1871.

Mayo, A.M., Chang, B.L., \& Omery, A. (2002). Use of protocols and guidelines by telephone nurses. Clinical Nursing Research, 11(2), 204-219.

Miaskowski, C., Dodd, M., \& Lee, K. (2004). Symptom clusters: The new frontier in symptom management research. Journal of the National Cancer Institute, Monographs, (32), 17-21.

Molassiotis, A., Wengstrom, Y., \& Kearney, N. (2010). Symptom cluster patterns during the first year after diagnosis with cancer. Journal of Pain \& Symptom Management, 39(5), 847-858.

Richard, M.L., Parmar, M.P., Calestagne, P.P., \& McVey, L. (2010). Seeking patient feedback: An important dimension of quality in cancer care. Journal of Nursing Care Quality, 25(4), 344-351.

Stacey, D., Bakker, D., Green, E., Zanchetta, M., \& Conlon, M. (2007). Ambulatory oncology nursing telephone services: A provincial survey. Canadian Oncology Nursing Journal, 17(4), 186(e1-5).

Stacey, D., Graham, I., O'Connor, A.M., \& Pomey, M.P. (2005). Barriers and facilitators influencing call centre nurses' decisions support for callers facing values-sensitive decisions: A mixed methods study. Worldviews on Evidence-Based Nursing, 2(4), 184-195.

Stacey, D., Noorani, H., Fisher, A., Robinson, D., Joyce, J., \& Pong, R. (2003). Telephone triage services: Systematic review of the literature and survey of Canadian call centres program. Ottawa: Canadian Coordinating Office of Health Technology Assessment.

Wilson, R., \& Hubert, J. (2002). Resurfacing the care in nursing by telephone: Lessons from ambulatory oncology. Nursing Outlook, 50(4), 160-164. 